\title{
Proceeding
}

Supplementary Issue: Winter Conferences of Sports Science. Costa Blanca Sports Science Events, 24 April 2020. Alicante, Spain.

\section{The effectiveness of motor activity on psychomotor development in school-aged children}

\author{
MATTEO ABATE, LUCIA PALLONETTO , CARMEN PALUMBO \\ Department of Human Sciences, Philosophy and Education, University of Salerno, Italy
}

\begin{abstract}
In continuity with research on learning through mirror neurons (Rizzolatti G., Sinigaglia C. 2005), with a constructivist conviction (Bellantonio, 2014) it is stated that sports practice, as an instrument that exposes the person to multiple and different motor stimuli and perceptive is an effective medium for psychomotor development. In addition to promoting physical growth and organic development, motor skills also promote the growth of learning. It is no coincidence that psychomotor activity educates and realizes the whole of the person and constitutes an important means of interacting with it through movement. (Lapierre, A., 2001) The research group from Salerno conducted a field research involving a sample of 281 children between 6 and 8 years old, who were given the APCM-2 protocol (Sabbadini, 2005) and who were divided into three categories thanks to a survey completed through questionnaires: children who practice motor activity, children who do not practice motor activity, children who have not returned the questionnaire (group that is supposed to be mixed). It has emerged that participation in a motor activity produces positive effects on the development of the motor pattern and therefore of the person as a whole, achieving homogeneously higher scores on average than the other groups. Therefore, motor activities in school age represent a facilitator in psychomotor development as the stimulation of fundamental motor skills helps children in recreational activities by allowing them to manipulate their environmental conditions and control their own bodies. (Goodway, 2003).
\end{abstract}

Keywords: APCM-2; Motor activity; Psychomotor development.

\section{Cite this article as:}

Abate, M., Pallonetto, L., \& Palumbo, C. (2020). The effectiveness of motor activity on psychomotor development in school-aged children. Journal of Human Sport and Exercise, 15(2proc), S222-S231. doi:https://doi.org/10.14198/jhse.2020.15.Proc2.13

Corresponding author. Department of Human Sciences, Philosophy and Education, University of Salerno, Italy.

E-mail: Ipallonetto@unisa.it

Supplementary Issue: Winter Conferences of Sports Science. Costa Blanca Sports Science Events, 24 April 2020. Alicante, Spain.

JOURNAL OF HUMAN SPORT \& EXERCISE ISSN 1988-5202

(c) Faculty of Education. University of Alicante

doi:10.14198/jhse.2020.15.Proc2.13 


\section{INTRODUCTION}

Psychomotor development refers to changes in a child's cognitive, emotional, motor and social skills, from the beginning of life to adolescence. Science has tried to interpret with different models the origins of human behaviour and the changes in development observed over time, as well as the individual and contextual factors that could guide the development of the child. However, no single theory has managed to understand all the many aspects of child development, since they are all determined to form the complex puzzle of child development.

Certainly, there are some conflicting theories, but in truth for the majority many of the information that they take into consideration are, indeed, complementary to each other. Therefore, this concordance leads to the configuration of a common and typical psychomotor development of the child, which ensures that the relative theories and models can be usefully used for clinical practice, especially in identifying the disorders and the ways in which these can be treated.

In continuity with research on learning through mirror neurons (Rizzolatti, Fogassi and Gallese, 2001), with constructivist conviction (Bellantonio, 2014) it is stated that sports practice, as an instrument that exposes the person to multiple and different motor stimuli and perceptual is an effective medium for psychomotor development.

One of the common data is that the infant's brain is equipped with a mirror neuron system (Casile A., Caggiano V., Ferrari P., 2011), which is influenced by factors, both internal and external, both before and after conception. and which have the ability to make epigenetic changes that affect brain neuroplasticity. These changes occur to allow adaptation to the environment and can take place as neuronal networks have the ability to perform multitasking activities (Ferrari PF., Rizzolatti G., 2014), or have various functions that allow visual-sensorial integration with motor coding.

In other words, neuronal networks in young people are capable of understanding actions and intentions at the same time as the brain draws up the visual-auditory map of the activity, our motor representation of the same activity (Rizzolatti et al., 2001). Rizzolatti et al. describe the mirror neuron system as a group of neurons located in the ventral premotor cortex and lower parietal lobule; a system stimulated by our own activities and by the observation of similar activities done by others, with the aim of facilitating the integration of ourselves with the environment (Rizzolatti G., Fabbri-Destro M., Cattaneo L., 2009).

During the neonatal period, the functions of the mirror neurons can be noted, observing the movements of the new-born's lips as a response (still unconscious) as a response to the stimuli of the parents (Simpson EA., Murray L., Paukner A., Ferrari PF., 2014). This imitation is remembered by the child, so it is not a reflection and allows the improvement of the interaction with the parents with each new stimulus (Simpson EA., Fox NA., Tramacere A., Ferrari PF., 2014). During growth, the child deconstructs the previous activities and repeats them to achieve a new goal, this happens inadvertently, until finally he learns to relate them to the environment that surrounds him. So, when more stimuli are provided to the child, feedback occurs that changes the behaviour. Gradually he spontaneously learns to adapt the speed of his movements and dexterity (fine motor skills) (Woodward AL., Gerson SA., 2014).

The development of motor skills also influences that of perceptive abilities and derives from the information that he receives with respect to a future response that comes from adults; therefore, the mirror neuron system is powered by the observation of actions. Also, according to Simpson et al., The observation of imitation 
behaviour in the new-born is extremely important in psychomotor development. Since mirror neurons depend on the actions of others, they appear to be a key element (Hamilton AF., 2013) to stimulate new capacities. So, when the neuron system observes something new, picks it up from the old memory, discards the new or learns it, which makes learning or imitating activities easier (Buccino G., Vogt S., Ritzl A., Fink GR., Zilles K., Freund HJ., et al., 2004). Physical training is fundamental for the development of children (Timmons, Naylor \& Pfeiffer, 2007; Trudeau \& Shephard, 2008), in fact, through motor experience, the self-awareness of children and their awareness are manifested and exist in relation to the world surrounding. We can say, therefore, that psychomotor development can be defined as the progressive acquisition of skills concerning both mental and motor activities. In addition to promoting physical growth and organic development, motor skills also promote the growth of learning. It is no coincidence that psychomotor activity educates and realizes the whole of the person and constitutes an important means of interacting with it through movement. (Lapierre A., 2001). The latter are a vital condition for a child to learn the teaching dedicated to him, to stimulate thought and creativity and therefore guarantee a better academic performance (Fisher et al., 2005; Stodden et al., 2008).

For the development of essential motor skills (Gallahue \& Donnely, 2003) the most important period is that of early childhood, in fact already in preschool children are naturally curious and willing to explore especially through play or suitably stimulated to play a physical activity. Although schools and kindergartens offer the ideal environment for these moments of pure discovery, it is highly recommended that parents, teachers and educators be trained for this purpose and create additional learning opportunities to experiment with alternative motor practices in places that are suitable and equipped with specialized materials. (Venetsanou \& Kambas, 2010). In summary, it is necessary that awareness is spread among all colours that deal with childhood in different ways, that physical activity is fundamental for the normal growth and psychomotor development of children.

\section{MATERIAL AND METHODS}

The goal of this research is to evaluate the effectiveness of sports in school age. The APCM-2 test (Sabbadini L., 2015) was used for the evaluation of praxic and motor-coordinative skills. Given its practical application, the protocol in question was used in a chaotic environment, as can often be the case at school, providing the opportunity to show children the items to be carried out (Bertoldo N., Siravegna D., Pacilli C., Gliozzi G., Bazzao D., 2008). The data submitted to the research were assessed through quantitative and qualitative surveys, the method used is therefore mixed. The qualitative information comes from the administration, to the parents, of the questionnaire (comprising a section normally attached to the APCM-2 test, used for the administration, and another session created to obtain information on sports habits), thanks to which it was possible to detect possible presence of disorders or particular complications on pregnancy, problems at birth or in the first years of life (allergies, ear infections etc...), type of nutrition, correct chewing, difficulty in sleeping, respiratory coordination, posture in play and walking, prehension, diction and sports habits. The quantitative information, on the other hand, comes from the results obtained by the children in the administration of the tests provided for by the test, which, in particular, are divided into two assessment areas: Movement patterns (which include the Balance, Oculomotion, Sequence scales, and hand and finger movements ) and Adaptive Cognitive Functions or Praxies (Dynamic Coordination, Grapho-Motor Skills, Manual Skills, Symbolic Gestures and Praxic-Constructive Skills).

During the administration phase, carried out in the field, the control exercised by the examiners was maximum, the administration of the test took place within the same school buildings but in environments previously prepared for the same, structuring it according to the times and spaces given to available from 
school managers. The environment was divided into 3 spaces, in each of which, the children would have performed different Items. The tests in each space were matched for the similarity of the necessary material and each space was entrusted to an examiner who proposed the same tests with each child for each class shift. The class group was further divided into 3 subgroups, one per examiner. At the end of the tests prepared for each space, the children were asked to move and change examiner thus determining that, with two movements in the three spaces, the children completed the execution of the entire test.

This criterion was used to make the administration less contaminated as possible by factors such as: distraction, boredom, anxiety, haste and stress that would have compromised the children's performance in performing the test and the examiners in producing a coherent assessment. At the end of the administration each examiner was in possession of a part of the results obtained by each child, the results were then compared, combined and reported on an Excel file.

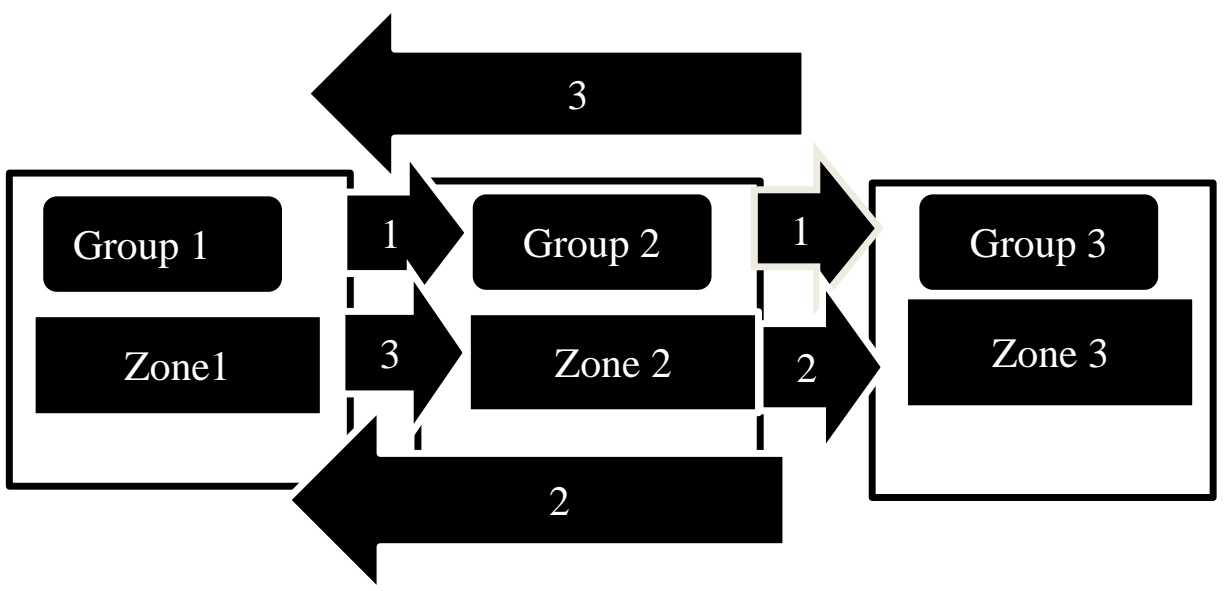

Note: Arrows indicate the movement of the groups.

Figure 1. Division of spaces. In this schema is indicated the division of spaces.

During the administration, the results obtained by the children in the different tests were noted on special tables created for the administration. All this has been achieved in order to manage the administration in an ecological logic by minimizing the confusion that can be created within the school environment.

Table 1. Example of table for catch the data during the somministration.

\begin{tabular}{|l|l|l|l|l|l|}
\hline & $\begin{array}{l}\text { Walk toward an } \\
\text { objective }\end{array}$ & $\begin{array}{l}\text { Kick a stopped ball with a } \\
\text { little bit running }\end{array}$ & $\begin{array}{l}\text { Run and to kick a } \\
\text { moving ball }\end{array}$ & $\begin{array}{l}\text { Jump an } \\
\text { obstacle }\end{array}$ & TOTAL \\
\hline 1 1) & & & & & \\
\hline 2 ) & & & & & \\
\hline
\end{tabular}

Note: For each evaluation compartment there are a different table. Horizontally there put the different exercises, vertically the name of the children with the his/her number.

\section{The sample}

The sample is made up of children aged between 6.1 and 8 years. The administration took place within the public school, considering it the most suitable place given the number of children it hosts and the heterogeneity it guarantees, and amounts to 281 children in its entirety. 
It was possible to have information on the frequency of sports for 172 children, as not all of them returned the questionnaire. Therefore, it was possible to divide the data into three categories divided as follows:

- Data in reference to children practicing sports (81 children),

- Data in reference to children not practicing sports (91 children),

- Data referring to children who did not submit the questionnaire (109 children and a mixed group is supposed).

\section{Tools}

For the investigation, the following tools were identified:

- Information questionnaire (addressed to the parents of the children involved). The questionnaire is the one that is normally attached to the Apcm-2 test to which, however, a section has been added concerning the possible sports practice carried out by the child.

- Evaluation test of praxic skills and motor coordination (APCM-2) (Sabbadini, 2015).

The material needed for the test is easy to find and produce, which can be consulted in the APCM-2 test manual.

Administration requires:

- Scotch pape,

- Ball Blank sheets (A4 size),

- Pencil,

- Rigid cardboard,

- Scissors,

- Cloth bag filled with beans,

- Cylinder with a diameter of about $30 \mathrm{~cm}$ (for example a wastepaper basket),

- Pencil sharpener,

- $1.5 \mathrm{mt}$ rope (to be used as an obstacle),

- Thin rope (with which to make the knots to be untied),

- Labels large enough to legibly write the name, surname and number assigned to the child.

\section{Research plan}

The APCM-2 test allows to obtain information and data on different areas of investigation, it was not considered appropriate to compare the individual areas of investigation with each other, because there is not enough information to be able to detect correspondences between the results. For this reason, the data was considered in its entirety consistently with a holistic view of individuals. Finally, the collected data were processed through Excel and conclusions were drawn from them.

\section{Data emerged}

It was initially questioned whether to eliminate the extreme values or not, thus avoiding contamination, also due to a few cases that could have altered the overall group count, therefore it was decided to consider all the available values. The outlier values were however researched, and already dividing the entirety of the results into quartiles, it can be observed that no child who practices sports ranks neither among the slightly anomalous values (inner fence), nor among the extremely anomalous ones (outer fence).

Before any other analysis we can say that:

- $5.5 \%$ of children who do not play sports have scored low enough to be considered abnormal, 
- $0 \%$ of children doing sports scored low enough to be considered abnormal,

- $4.6 \%$ of the children who did not return the questionnaire scored low enough to be considered abnormal (however, we do not know how many of these play sports).

For each Item carried out, the children were assigned a score of 0 points in the event that they failed to perform the required test, 1 point in the event that the performance was partial, and 2 points in the event that the test is completed without error. At the end of the administration the situation detected is that represented by the following tables.

Table 2. Scorer's table of children that play sport.

\begin{tabular}{lll}
\hline Sample & Media & Variance \\
\hline First Classes & 93.02703 & 102.6381 \\
Second Classes & 101.7273 & 68.01691 \\
All the Pupils & 97.75309 & 101.7633 \\
\hline
\end{tabular}

Table 3. Scorer's table of children that don't play sport.

\begin{tabular}{lll}
\hline Sample & Media & Variance \\
\hline First Classes & 87.89286 & 135.0429 \\
Second Classes & 96.31429 & 161.6924 \\
All the Pupils & 91.13187 & 160.5824 \\
\hline
\end{tabular}

Table 4. Scorer's table of children that haven't given back the questionnaire.

\begin{tabular}{lll}
\hline Sample & Media & Variance \\
\hline First Classes & 88.61905 & 155.7235 \\
Second Classes & 94.86957 & 340.8715 \\
All the Pupils & 91.25688 & 241.0445 \\
\hline
\end{tabular}

\section{RESULTS}

Based on the information learned, the children were grouped into three groups and the mean and variance were then obtained from the results obtained. From what emerged as expected sport practice seems to have positive effects on the development of the motor pattern, but to have a broader perspective it is necessary to compare the values of the 3 investigation groups in order to perform a more precise analysis and draw the right meaning:

Table 5. Media comparison table.

\begin{tabular}{lccc}
\hline Average scores & Sport & No Sport & No Quest \\
\hline First classes & 93 & 87.8 & 88.6 \\
Second classes & 101.7 & 96.3 & 94.8 \\
All the Pupils & 97.7 & 91.1 & 91.2 \\
\hline
\end{tabular}

Comparing the averages, it is clear that:

- The subgroup of children who play sports tends, of the three, to be the one with the highest average score,

- Observing all the children, the group of children who did not submit the questionnaire, at times, reached a significantly higher average score, compared to those who certainly do not practice sports. 
It is reasonable to assume that this subgroup is made up of practicing and non-practicing people and the fact that the average score is between the other two subgroups in a random and non-unitary way, could be determined by the presence or absence of sportsmen in the group,

- In the three subgroups there is a considerable improvement in psychomotor development between the first and second year of primary school, this data confirms that psychomotor development is consolidating around 7 years.

Table 6. Variance comparison table.

\begin{tabular}{lccc}
\hline Variance comparison table & Sport & No Sport & No Quest \\
\hline First Classes & 102.6 & 135 & 155.7 \\
Second Classes & 68 & 161.6 & 340.8 \\
All the Pupils & 101.7 & 160.5 & 241 \\
\hline
\end{tabular}

The variance index tends to be lower among children who play sports than the other two subgroups. From this data it is clear that there is a homogeneous psychomotor development, also having the test a maximum score which all groups approach. A lower variance index means that, basically, children who play sports and who had lower scores, however, had scores closer to the average value, compared to children with low scores belonging to the other subgroups. In the total count of children, it is noted that the group of children, who did not return the questionnaire, has the highest variance index, compared to the other subgroups. Assuming that it is considered reasonable to think that this subgroup is a mixed group, and therefore, composed of children who play sports and children who do not practice sports, the fact that it presents greater heterogeneity could be due to the presence in this group of children who would be had to belong to two different groups. There is no evidence to affirm this, and in fact this observation will not be taken into account in the final considerations, however it was considered appropriate to highlight this possibility given the anomaly of the data.

\section{Graphical comparison of the trend}

Being composed of different units, the graphs to be compared with each other are representative of the percentage of children, for each group, who obtained a certain result in the test. In order to appreciate the trend, it was considered appropriate to identify the number of children included for each cluster of three points (since between a child who obtained a score of 90 and one who obtained a score of 91 there are no substantial differences, the neighbouring scores have been grouped in the same group, every 3 points, which will be identified by the number of children it groups).

Analysing the graph, having the three different curves is already significant in itself. In detail, however, the curves show that a child who practices sports, is more likely to have better psychomotor development. The ascending phase of the curve, which indicates the group of children doing sports, is fairly linear compared to the others, the trend towards the average value is found mostly in the group of children who play sports, the other two groups on the contrary present numerous peaks. In the sports group the values are all close to the average value, this, precisely because sport has allowed us to favour where necessary and where there were shortcomings in development, resulting in a more homogeneous condition. 


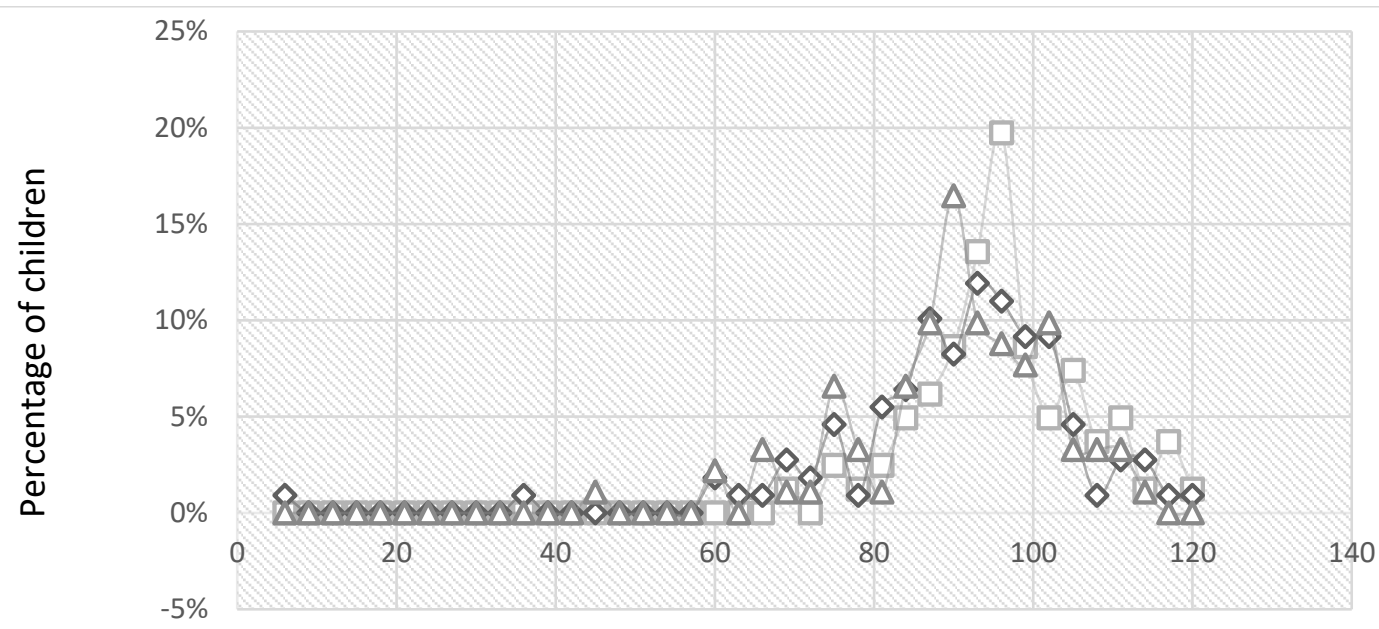

Score in the test APCM-2

- Pupils that play sport

$\diamond$ Pupils that have't given back the questionnaire

$\Delta$ Pupils that don't play nothing

Figure 2. Graphic comparison of the trends in various groups, on the $X$ there are the scores in the test APCM2 , on the $Y$ there are the percentages of the children (relative to their cluster).

\section{CONCLUSION}

At the beginning of the research, one wondered about the real possibility of positively intervening on psychomotor development, through sports. According to the results of the administration, it was possible to show that the group of children who play sports is the group that has obtained better results on average and which, at the same time, is more uniform than the others.

Furthermore, what was interesting to bring out (and which was not expected) is the "corrective-auxiliary" function of sports (it should be remembered that the administration was carried out in the school-public context and without applying particular selective criteria if not that sports had been practiced for at least a month). Motor activity proves to be "corrective", in that it allows you to improve your ability and dexterity, and "auxiliary" in that it allows you to help those who do not have a psychomotor development corresponding to or appropriate to their age. It was confirmed that without having practiced sports previously, some of the children would have obtained lower scores and that thanks to the training, however, they managed to approach an average standard even when they did not obtain particularly high scores. Beyond the assumptions, it is clear that the group of children who play sports has a more homogeneous psychomotor development than the other groups and that sport has been a valid auxiliary tool to allow individual young individuals to develop the basic psychomotor functions that allow you to live peacefully in society. Thinking in a future perspective, the sport will allow today's children to be, tomorrow, adults with greater possibilities to relate to themselves and the surrounding environment, thus developing a greater mastery of the body. Therefore, this article is intended to demonstrate the main role of movement in the child's growth process and how physical education, as a school subject, can make a fundamental contribution to achieving cognitive and motor goals. Although mind and body have long been considered two separate entities in both medical 
and educational settings, the movement proves to be an «efficient way for students to learn and remember content» (Lengley \& Kuczala, 2010, p. 8).

According to the author Ratey (2000), movement is the «miracle of growth»: it can promote the growth of children from a variety of perspectives. We can speak of learning through practical activities, of manipulation of ideas and objects, of body language transmitted through gestures and posture, also through facial expressions, in order to show the connection between body and mind. But what we have tried to emphasize so far is the importance of physical activity in the evolution of the child's psychomotor development. Therefore, the motto «I move, therefore I am » (Seitz, 1993) should be a reminder of the centrality of the movement in the school curriculum. Finally, although it is recognized that physical education is important for the student and for the related benefits such as, for example, the development of self-regulation, autonomy and self-esteem - in addition to promoting health and well-being and development of motor and cognitive skills - the ultimate goal must be to give it a more central role in primary education and allow it, through greater attention in the training of both teachers and students, to lay the foundations for subsequent development and more mature learning.

\section{AUTHOR CONTRIBUTIONS}

Abate M. Graduated in Motor and Sport Evaluation Sciences and Analysis and Design Techniques for Disabled Sports. He is the author of the research. Pallonetto L. Ph.D. Student in Didactic Corporealities, Technology and Inclusion, at the Department of Human Sciences, Philosophy and Education of the University of Salerno, research fields: "Material and Methods". Palumbo C., Associate professor of Methods and Didactics of Motor Activities (SSD M-EDF / 01), at the Department of Human Sciences, Philosophy and Education of the University of Salerno, as Scientific Curator. Research field: "Introduction" and "Conclusion".

\section{REFERENCES}

Axeti G., Gissis I., Vrabas I., Grouios G., Komsis G., \& Komsis S. (2017). Assessment of kinematic characteristics of preschoolers' gait during the implementation of an intervention training program. Journal of Human Sport and Exercise, 12(4), pp. 1298-1309. https://doi.org/10.14198/jhse.2017.124.16

Bellantonio S. (2014). Sport e adolescenza. L'educazione come promozione delle risorse. Milano: Franco Angeli, p. 87.

Bertoldo N., Siravegna D., Pacilli C., Gliozzi G., Bazzao D. (2008). La valutazione della disprassia evolutiva: sperimentazione e confronto fra le scale maggiormente in uso in età evolutiva. Giornale di Neuropsichiatria dell'Età Evolutiva.

Buccino G., Vogt S., Ritzl A., Fink GR., Zilles K., Freund HJ., et al. (2004). Neural circuits underlying imitation learning of hand actions: an event-related fMRI study. Neuron. Apr 22;42(2):pp.323-34. https://doi.org/10.1016/s0896-6273(04)00181-3

Casile A, Caggiano V, Ferrari PF. (2011). The mirror neuron system: a fresh view. Neuroscientist. Oct;17(5):pp.524-38. https://doi.org/10.1177/1073858410392239

Čillík, I., \& Willwéber, T. (2018). Influence of an exercise programme on level of coordination in children aged 6 to 7 . Journal of Human Sport and Exercise, 13(2), 455-465. https://doi.org/10.14198/ihse.2018.132.14

Ferrari PF., Rizzolatti G. (2014). Mirror neuron research: the past and the future. Philos Trans R Soc Lond B Biol Sci. 1 Apr 28;369(1644):20130169. https://doi.org/10.1098/rstb.2013.0169 
Fionda B., (2005). Application and experimentation of the APCM Protocol in a sample of children with Specific Language Disorder: correlation between DSL and dyspraxia. In: Protocol for the evaluation of Praxic Skills and Motor Coordination APCM. Rehabilitation Methodologies in Speech Therapy, vol 13. Springer, Milan, pp. 101-108.

Fisher A., Reilly J., Kelly L., Montgomery C., Williamson A., Paton J. (2005). Fundamental movement skills and habitual physical activity in young children. Medicine and Science in Sports and Exercise, 37, pp. 684-688. https://doi.org/10.1249/01.mss.0000159138.48107.7d

Gallahue, D., \& Donnelly F. (2003). Developmental physical education for all children. Champaign: Human Kinetics.

Hamilton AF. (2013). Reflecting on the mirror neuron system in autism: a systematic review of current theories. Dev Cogn Neurosci. Jan; 3:pp.91-105.

Lengel T., Kuczala M. (2010). The kinesthetic classroom Teaching and through movement. Thousand Oaks, CA Corwin Press.

Lapierre A., (2001). From relational psychomotor skills to the bodily analysis of the relationship. Rome: Armando Editore, p.20.

Lago-Rodríguez A., Cheeran B., Koch G., Hortobagy T., Fernandez del Olmo M. (2014). The role of mirror neurons in observational motor learning: an integrative review, European Journal of Human Movement.

Le Bel RM., Pineda JA., Sharma A. (2009). Motor-auditory-visual integration: The role of the human mirror neuron system in communication and communication disorders. J Commun Disord. JulAug;42(4): pp.299-304. https://doi.org/10.1016/j.jcomdis.2009.03.011

Rizzolatti G., Fabbri-Destro M., Cattaneo L. (2009), Mirror neurons and their clinical relevance. Nat Clin Pract Neurol. Jan;5(1): pp.24-34. https://doi.org/10.1038/ncpneuro0990

Rizzolatti G., Sinigaglia C. (2005). So quello che fai. II cervello che agisce e i neuroni a specchio. Milano: Raffaello Cortina Editore.

Sabbadini L. (2015). Manuale APCM-2, Abilità Prassiche e della coordinazione motoria $2^{\circ}$ edizione. Firenze: Hogrefe Editore.

Seitz J. (1993). I move.... therefore I am. Psychology Today, 26, pp.50-55.

Sgambelluri R., Pistoni M. (2017). The podalic proprioception channel to increase motor skills in blind children: Operational applications. Journal of Human Sport and Exercise, 12(3), pp.753-759. https://doi.org/10.14198/ihse.2017.123.19

Simpson EA., Fox NA., Tramacere A., Ferrari PF. (2014). Neonatal imitation and an epigenetic account of mirror neuron development. Behav Brain Sci. Apr;37(2):220. https://doi.org/10.1017/s0140525x13002495

Simpson EA., Murray L., Paukner A., Ferrari PF. (2014). The mirror neuron system as revealed through neonatal imitation: presence from birth, predictive power and evidence of plasticity. Philos Trans $R$ Soc Lond B Biol Sci. Apr 28;369(1644):20130289. https://doi.org/10.1098/rstb.2013.0289

Timmons B.W., Naylor P-J., Pfeiffer K. A. (2007). Physical activity for preschool children-how much and how? Applied Physiology, Nutrition and Metabolism, 32(Suppl), pp.S122-134.

Venetsanou F., Kambas A. (2010). Environmental factors affecting preschooler's motor development. Early Childhood Education Journal, 37, pp.319-327. https://doi.org/10.1007/s10643-009-0350-z

Woodward AL., Gerson SA. (2014), Mirroring and the development of action understanding. Philos Trans R Soc Lond B Biol Sci. Apr 28;369(1644):201301.

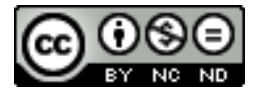

This work is licensed under a Attribution-NonCommercial-NoDerivatives 4.0 International (CC BY-NC-ND 4.0). 